\title{
Production line for hepatocytes
}

DOI:

10.1038/nrd2427
Human hepatocytes have a key role in studies of the metabolism and toxicity of candidate drugs, but reliable, high-quality sources of these cells are lacking. Reporting in Nature Biotechnology, Azuma and colleagues have now created immunodeficient mice that can be highly engrafted with human hepatocytes, providing a robust platform for their production.

Previous efforts to expand human hepatocytes in rodents have been based on immunosuppressed transgenic mice in which expression

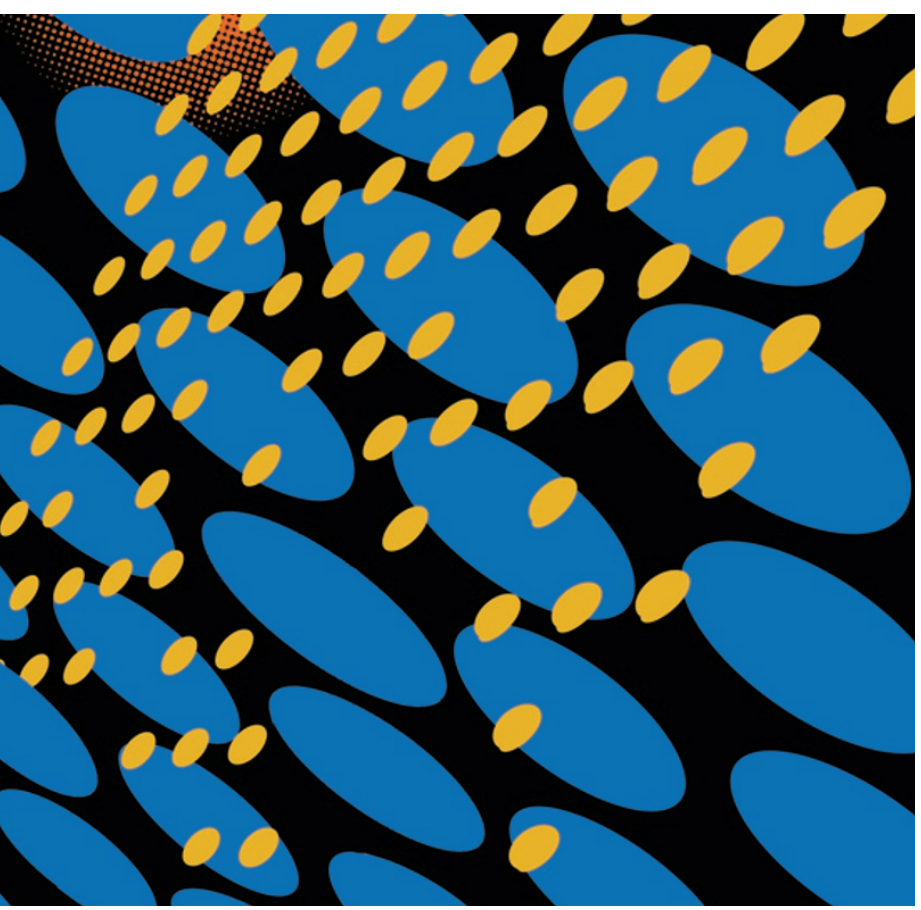

of the serine protease urokinase plasminogen activator (uPA) is used to cause major liver damage before transplantation of hepatocytes, which then proliferate. However, despite achieving engraftment levels of up to $70 \%$ in these models, problems such as breeding difficulties and inability to expand engrafted hepatocytes by serial transplantation have limited the use of uPA transgenic models.

With the aim of addressing these problems, the authors crossed mice that were deficient in the tyrosine catabolic enzyme fumarylacetoacetate hydrolase (FAH) onto the immuno-

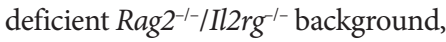
to create $\mathrm{Fah}^{-1-} / \mathrm{Rag}^{-1-} / \mathrm{Il} \mathrm{rg}^{-1-}$ (FRG) mice. Owing to the FAH deficiency, liver damage in this model can be controlled by administration (to prevent accumulation of toxic tyrosine catabolic intermediates) and withdrawal (to accumulate toxins that cause liver injury) of the drug 2-(2-nitro-4-trifluoromethylbenzoyl)-1,3-cyclohexanedione (NTBC).

FRG mice did not readily accept human hepatocyte xenografts, but successful engraftment was achieved by incorporating an aspect of the UPA transgenic model: intravenous injection of a uPA-expressing adenovirus, which had previously been shown to enhance hepatocyte engraftment. This was then followed by intrasplenic injection of $1 \times 10^{6}$ human hepatocytes and gradual withdrawal of NTBC.
Human hepatocyte repopulation was high (30-90\%) in $~ 16 \%$ of the mice.

The mass of highly engrafted mice stabilized during NTBC withdrawal, suggesting that the transplanted human cells replaced the function of the diseased $\mathrm{Fah}^{-/-}$recipient hepatocytes. FAH immunostaining confirmed that $>70 \%$ of the hepatocytes were FAH-positive and reverse-transcription PCR confirmed that they expressed mature hepatocyte-specific genes including human albumin.

Serial transplantation of hepatocytes into secondary, tertiary and quaternary recipients resulted in an in vivo expansion of at least 150-fold in each round. And, in contrast to previous uPA models, transplantation into FRG mice can be performed at any age, and the mutant mice are fully fertile when continuously administered with NTBC.

Finally, to characterize the engrafted human hepatocytes, cultures of isolated hepatocytes were subjected to standard drug metabolism assays and were found to be equivalent to cultured normal adult human hepatocytes. FRG mice could therefore provide a reliable source of human hepatocytes for drug metabolism and toxicity studies, as well as for studies on liver diseases such as hepatitis C.

Bethan Hughes

ORIGINAL RESEARCH PAPER Azuma, H. et al. Robust expansion of human hepatocytes in $\mathrm{Fah}^{-1}$ /Rag2-'//I2rg-/- mice. Nature Biotech. 25 , 903-910 (2007) 